\title{
Analysis of 256 Cases of Classic Fever of Unknown Origin
}

\author{
Masashi Yamanouchi, Yuki Uehara, Hirohide Yokokawa, Tomohiro Hosoda, \\ Yukiko Watanabe, Takayoshi Shiga, Akihiro Inui, Yukiko Otsuki, Kazutoshi Fujibayashi, \\ Hiroshi Isonuma and Toshio Naito
}

\begin{abstract}
Objective The causes of fever of unknown origin (FUO) vary depending on the region and time period. We herein present a study of patients with classic FUO where we investigated differences based on patient background factors, such as age and causative diseases, and changes that have occurred over time.

Methods We extracted and analyzed data from the medical records of 256 patients $\geq 18$ years old who met the criteria for classic FUO and were hospitalized between August, 1994 and December, 2012.

Results The median age of the patients was 55 years (range: 18-94 years). The cause of FUO was infection in $27.7 \%$ of the patients $(n=71)$, non-infectious inflammatory disease (NIID) in $18.4 \%$ (47), malignancy in $10.2 \%$ (26), other in $14.8 \%$ (38), and unknown in $28.9 \%$ (74). The most common single cause was human immunodeficiency virus (HIV)/acquired immunodeficiency syndrome (AIDS) $(n=17)$. NIID and malignancy were more common in patients $\geq 65$ years old than in patients $<65$ years old. During 2004-2012, compared to 1994-2003, infections and "other" causes were decreased, whereas NIID, malignancy, and unknown causes were increased.

Conclusion FUO associated with HIV/AIDS is increasing in Japan. In addition, as in previous studies in Japan and overseas, our study showed that the number of patients in whom the cause of FUO remains unknown is increasing and exceeds $20 \%$ of all cases. The present study identified diseases that should be considered in the differential diagnosis of FUO, providing useful information for the future diagnosis and treatment of FUO.
\end{abstract}

Key words: human immunodeficiency virus/acquired immunodeficiency syndrome, malignant lymphoma, image studies

(Intern Med 53: 2471-2475, 2014)

(DOI: 10.2169/internalmedicine.53.2218)

\section{Introduction}

Fever of unknown origin (FUO) was defined by Petersdorf and Beeson in 1961 as a fever of $\geq 38.3^{\circ} \mathrm{C}$ that lasts for $\geq 3$ weeks and remains undiagnosed even after 1 week of inpatient evaluation (1). In 1991, Durack and Street further classified FUO into classic FUO, nosocomial FUO, neutropenic FUO, and human immunodeficiency virus (HIV)associated FUO. In addition, the criterion of "undiagnosed after 1 week of inpatient evaluation" has been changed to "undiagnosed after 3 outpatient visits or 3 days of inpatient evaluation," so that it may also apply to outpatients (2).
FUO may be caused by many diseases that vary depending on the region and time period. The causes of FUO are generally considered to be infection, non-infectious inflammatory disease (NIID), and malignancy (1-9). In the existing literature, the causes of FUO have been classified into 5 categories: infection, NIID, malignancy, other, and unknown (5, 8-17). Studies on the causes of FUO have occasionally been reported in Japan, but the number of cases has been small, often representing regional studies limited to a single hospital (13-17). The first nationwide retrospective study of FUO in Japan has recently been reported (18). Pediatric case studies of FUO in Japan have also been collectively reported (19). However, accumulating cases of hospi- 
(n)

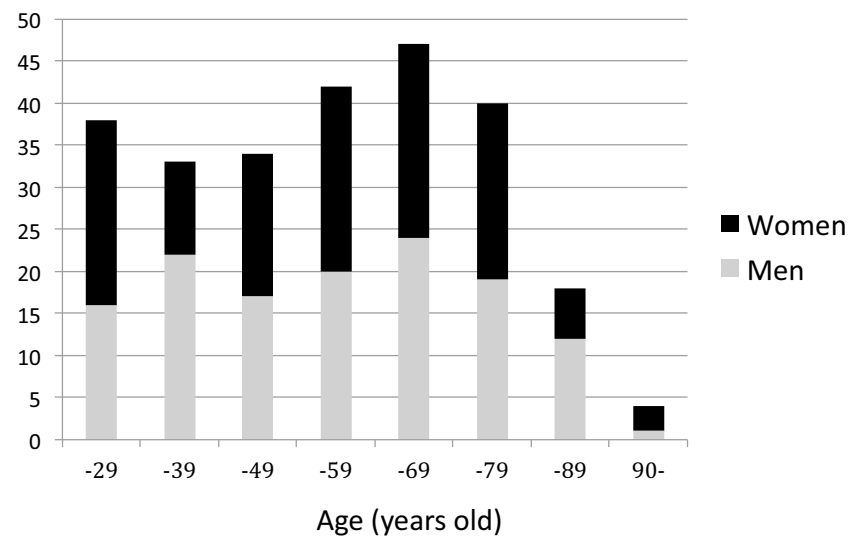

Figure 1. The age distribution of the patients.

talized patients meeting the criteria for classic FUO is not easy. Unless a hospital is mid-sized or larger and receives referrals from other hospitals for further testing, or unless there is collaboration with other institutions, acquiring a sufficient number of cases is difficult. Over the subsequent 10year period, the numbers of FUO patients referred for further testing or admitted to our hospital have increased (20). Therefore, the aim of the present study was to investigate the recent trends in classic FUO; specifically, we focused on the differences based on patient background factors, such as age and causative diseases, and changes that have occurred over time.

\section{Materials and Methods}

For the present single-institution retrospective observational study at a 1,020-bed university-affiliated hospital in Tokyo, data were extracted from the medical records of patients $\geq 18$ years old who met the criteria for classic FUO and were hospitalized between August 1, 1994 (when our department was established) and December 31, 2012. The patients treated in our hospital are referred from a wide geographic distribution throughout Japan (75.4\% from Tokyo, $22.1 \%$ from Eastern Japan other than Tokyo, and 2.7\% from Western Japan; $n=32,149$, data as of 2012). The criteria of Durack and Street for classic FUO were used in this study: fever $\geq 38^{\circ} \mathrm{C}$ at least twice over a $\geq 3$-week period, lack of a diagnosis after 3 outpatient visits or 3 days of hospitalization, and no diagnosis of HIV or immunodeficiency prior to the fever (2). The temperatures were measured as axillary temperature, which is often measured in Japan.

The causes of FUO were classified as infection, NIID, malignancy, other, and unknown based on the medical records by the physician in charge of each patient. All cases, in which the causes were only suspected, not confirmed, were classified as unknown. Because the categorizations of specific diseases sometime differ between studies, previous articles were used as references for the present study $(5,9,21)$.

The following data were compiled and analyzed in the
Table 1. The Causes of Classic FUO

\begin{tabular}{lc}
\hline \multicolumn{1}{c}{ Causes } & Number (\%) \\
\hline Infection & $\mathbf{7 1}(\mathbf{2 7 . 7 \% )}$ \\
HIV/AIDS & 17 \\
Infective endocarditis & 12 \\
Tuberculosis & 4 \\
Other & 38 \\
NIID & $\mathbf{4 7}(\mathbf{1 8 . 4 \% )}$ \\
Polymyalgia rheumatica & 10 \\
Adult Still's disease & 8 \\
Reactive arthritis & 5 \\
Behçet's disease & 4 \\
Other & 20 \\
Malignancy & $\mathbf{2 6}(\mathbf{1 0 . 2 \% )}$ \\
Malignant lymphoma & 12 \\
Other & 14 \\
Other & $\mathbf{3 8}(\mathbf{1 4 . 8 \% )}$ \\
Necrotizing lymphadenitis & 7 \\
Subacute thyroiditis & 5 \\
Other & 26 \\
Unknown & $\mathbf{7 4}(\mathbf{2 8 . 9 \% )}$ \\
\hline
\end{tabular}

HIV/AIDS: human immunodeficiency virus/acquired immunodeficiency syndrome, NIID: non-infectious inflammatory disease

present study:

$<1>$ The age group, sex, and causes of FUO $(\%)$ in all patients,

$<2>$ the differences in the causes of FUO (\%) between patients $\geq 65$ years old and $<65$ years old,

$<3>$ the differences in the causes of FUO (\%) between the periods 1994-2003 and 2004-2012, and

$<4>$ the number of cases requiring $\geq 150$ days for diagnosis from the time of fever onset.

\section{Results}

A total of 256 patients were enrolled in this study, including 131 men and 125 women, with a median age of 55 years (range: 18-94 years). The most frequent age group comprised patients in their 60s (Fig. 1). Patients $\geq 65$ years old accounted for $33.2 \%$ (85/256) of all patients. The cause of FUO was infection in $27.7 \%$ of patients $(n=71)$, NIID in $18.4 \%(n=47)$, malignancy in $10.2 \%(n=26)$, other in $14.8 \%$ $(\mathrm{n}=38)$, and unknown in $28.9 \%(\mathrm{n}=74)$. The most common single cause was HIV/acquired immunodeficiency syndrome (AIDS) $(n=17)$, accounting for $25 \%$ of all infections. Among these, 16 patients were $<65$ years old. The next leading causes were malignant lymphoma $(n=12)$ and infective endocarditis $(n=12)$; with these causes, 4 patients and 10 patients, respectively, were $<65$ years old. The fourth leading cause was polymyalgia rheumatica $(n=10) ; 7$ were $\geq 65$ years old (Table 1 ).

In patients $\geq 65$ years old, compared to those $<65$ years old, the percentages of infections $(21.7 \%$ vs. $30.6 \%)$ and other causes $(7.2 \%$ vs. $18.5 \%)$ were lower, whereas the per- 
Table 2. The Percentages of the Causes by Category in Patients $\geq 65$ Years Old and $<65$ Years Old

\begin{tabular}{lccc}
\hline & Total \% (n) & $\mathbf{2 6 5}$ years \% (n) & $<\mathbf{6 5}$ years \% (n) \\
\hline Infection & $27.7(71)$ & $21.7(18)$ & $30.6(53)$ \\
NIID & $18.4(47)$ & $27.7(23)$ & $13.9(24)$ \\
Malignancy & $10.2(26)$ & $14.5(12)$ & $8.1(14)$ \\
Other & $14.8(38)$ & $7.2(6)$ & $18.5(32)$ \\
Unknown & $28.9(74)$ & $28.9(24)$ & $28.9(50)$ \\
\hline Total & $100(256)$ & $100(83)$ & $100(173)$ \\
\hline
\end{tabular}

NIID: Non-infectious inflammatory disease

Table 3. The Cases Requiring $\geq 150$ Days for Diagnosis from the Time of Fever Onset

\begin{tabular}{cc}
\hline Causes & $\begin{array}{c}\text { Duration } \\
\text { (days) }\end{array}$ \\
\hline Adult Still's disease & 325 \\
HIV/AIDS & 253 \\
Malignant lymphoma & 250 \\
Takayasu's arteritis & 246 \\
Chronic active EBV infection & 243 \\
Malignant lymphoma & 185 \\
HIV/AIDS & 160 \\
Macroglobulinemia & 154 \\
Malignant lymphoma & 150 \\
\hline
\end{tabular}

EBV: Epstein-Barr virus, HIV/AIDS: human immunodeficiency virus/acquired immunodeficiency syndrome

centages of NIID (27.7\% vs. $13.9 \%)$ and malignancy $(14.5 \%$ vs. $8.1 \%)$ were higher. The percentage of unknown causes was the same (28.9\%) in both patients $<65$ years old and $\geq 65$ years old (Table 2). In 2004-2012, compared to 1994-2003, the percentages of NIID, malignancy, and unknown causes tended to increase (Fig. 2). Among the cases with a diagnosed cause, a definitive diagnosis was not achieved until $\geq 150$ days from fever onset in 9 patients. The longest time to diagnosis was 325 days in a patient with adult Still's disease (Table 3).

\section{Discussion}

The causes of classic FUO vary with the region and time period. In previous reports before the 1990s, infections were the leading cause, whereas NIID and unknown causes have increased more recently $(5,9)$. The data from the 1950 s to the early 1990s have been compiled, and unknown causes have accounted for $\geq 20 \%$ of cases since the 1990s $(22,23)$. Our study also found a high percentage $(28.9 \%)$ of unknown causes of FUO. One reason for this increase in unknown causes may be as follows: in a university hospital like ours, many patients who are referred from other hospitals for further evaluation of FUO have already undergone

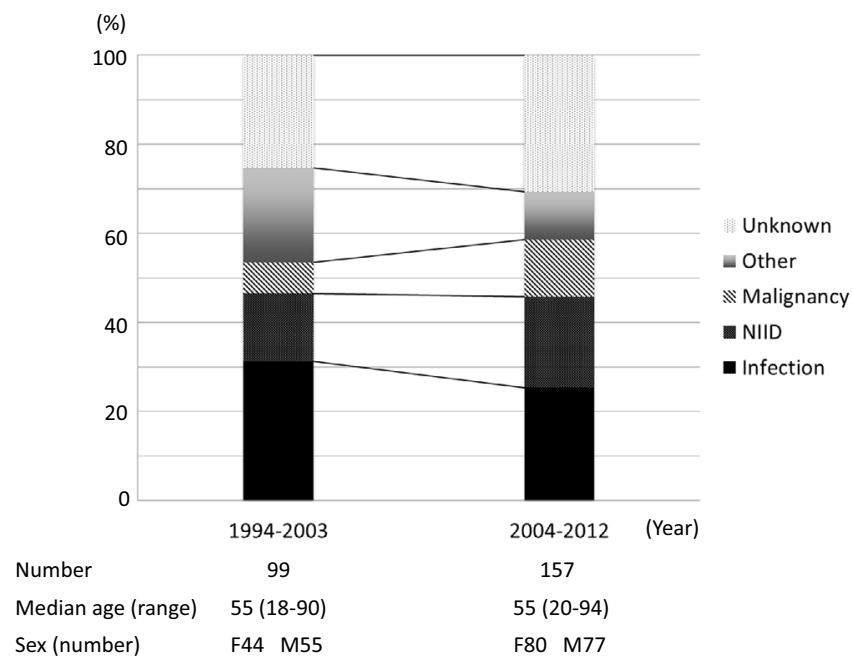

Figure 2. The percentages of the causes by category according to the time period. NIID: non-infectious inflammatory disease

routine testing by their local physician because of the wider availability of testing. The ratio of patients with FUO referred from other hospitals gradually increased from $59.6 \%$ in $1994-2003$ to $68.2 \%$ in $2004-2012$. There are, therefore, more cases where a diagnosis cannot be reached despite repeated testing.

The trends for some high-frequency cases are similar to findings from previous reports, but the increase in HIV/ AIDS is noteworthy. The number of HIV/AIDS patients in Japan is increasing. In 2012, there were 1,002 new cases of HIV infection and 447 patients with AIDS reported. Before 2012, a total of 14,706 cases of HIV infection and 6,719 AIDS patients had been reported. The number of newly infected persons increased from 1985 to 2008. Although there has been no subsequent increase, there has also not been any clear decrease (24). The number of these patients is expected to continue to increase in Japan, so HIV/AIDS must be taken into consideration as a potential cause of FUO.

Malignant lymphoma accounted for 12 of 26 cases of malignancy as a causative disease. In many cases, the symptoms are not always severe. Patients who present with fever or lymphadenopathy alone are often initially thought to have viral infections that are self-limiting, so more time may elapse before further invasive evaluation. With malignant lymphoma as a cause of FUO, a mean of 2 months between fever onset and diagnosis has been reported (25). In 3 of the 12 patients with malignant lymphoma in our study, the length of time required until a definitive diagnosis could be made was 150 days, 185 days, and 250 days, respectively.

A comparison between patients $\geq 65$ and $<65$ years old showed that infections were more common in patients $<65$ years old. This was influenced by the fact that this age group accounted for 16 of the 17 cases of HIV/AIDS and 10 of the 12 cases of infective endocarditis. Moreover, patients already diagnosed with HIV/AIDS at the time of fever development were excluded. The prevalence of NIID in pa- 
Table 4. Comparison of the Results of the Studies of Classic FUO in Japan

\begin{tabular}{|c|c|c|c|c|c|c|c|}
\hline Reference & Year & n & Infection & NIID & Malignancy & Other & Unknown \\
\hline 14 & $\begin{array}{l}1982- \\
1992\end{array}$ & 153 & $28.8 \%$ & $29.4 \%$ & $14.4 \%$ & $15.7 \%$ & $11.8 \%$ \\
\hline 13 & $\begin{array}{l}1986- \\
1992\end{array}$ & 80 & $53.7 \%$ & $16.2 \%$ & $8.8 \%$ & $3.8 \%$ & $17.5 \%$ \\
\hline 16 & $\begin{array}{l}2006- \\
2007\end{array}$ & 81 & $35.8 \%$ & $25.9 \%$ & $9.9 \%$ & \multicolumn{2}{|c|}{$28.4 \%$} \\
\hline 17 & 2011 & 56 & $21.4 \%$ & $35.7 \%$ & $7.1 \%$ & $12.5 \%$ & $23.2 \%$ \\
\hline 18 & 2011 & 121 & $23.1 \%$ & $30.6 \%$ & $10.7 \%$ & $12.4 \%$ & $23.1 \%$ \\
\hline Present study & $\begin{array}{l}1994- \\
2012\end{array}$ & 256 & $27.7 \%$ & $18.4 \%$ & $10.2 \%$ & $14.8 \%$ & $28.9 \%$ \\
\hline
\end{tabular}

NIID: non-infectious inflammatory disease

tients $\geq 65$ years old was greatly influenced by the ratio of polymyalgia rheumatica, which accounted for 7 of the 23 cases of NIID in patients $\geq 65$ years old. Epidemiologically, malignancy is more frequent among $\geq 65$ years old patients, and 8 of the 12 patients with malignant lymphoma were $\geq 65$ years old. The prevalence of "other" causes in patients $<65$ years old was influenced by the greater incidences of necrotizing lymphadenitis $(n=7)$ and subacute thyroiditis $(n=5)$ in these patients. The unknown causes accounted for a high percentage of FUO cases, $28.9 \%$ each in patients $<65$ years old and $\geq 65$ years old, without any bias toward age.

The elderly population is expected to continue increasing, and this change will be particularly dramatic in Japan compared to other countries. According to demographic studies, $25 \%$ of persons in Japan are $\geq 65$ years old and $40 \%$ of the population is expected to be $\geq 65$ years old by 2060 (26). The rates of diseases often seen in older adults, such as polymyalgia rheumatica and malignancy, will most likely continue to rise. Polymyalgia rheumatica, as in the past, still requires time for diagnosis because there are no specific antibody tests, symptoms take time to develop, and other diseases must be ruled out. Conversely, advances in imaging, such as positron emission tomography/computed tomography, and genetic testing may enable earlier diagnosis of NIID, such as temporal arteritis and Takayasu's arteritis, as well as malignancies.

A comparison of the 10-year period between 1994 and 2003 and the 9-year period between 2004 and 2012 showed trends in the causes of FUO based on time period. Age showed about the same distribution, and no significant biases were apparent with regard to sex. Infections and "other" causes decreased, whereas NIID, malignancy, and unknown causes increased. The trend in 2004-2012 was similar to that in the 1990 s in an overseas report (22). Recent reports about classic FUO in Japan compared to those before 2000 show an increase in the percentage of unknown causes, stated as $\geq 20 \%$ of cases in all reports. Our study showed a similar trend (Table 4). The decrease in infections is probably due to earlier diagnosis with imaging and ge- netic testing and reduced frequency of patients with tuberculosis. The increase in malignancies was influenced by a greater number of malignant lymphomas in 2004-2012.

In 9 of the 256 patients, $\geq 150$ days were required from the time of fever onset for diagnosis. The longest time was 325 days in a patient with adult Still's disease. That patient was a 48-year-old man who had fever with mild lymphadenopathy and joint pain. However, these symptoms did not occur together, so time was required before a diagnosis could be reached. The patient experienced joint pain, skin rash, and liver dysfunction approximately one month before diagnosis, which led to the diagnosis.

This study was a retrospective summary of cases, and each diagnosis was made by the treating physician at that specific time. Moreover, differences exist in regional and institutional features, and the fact that this is an urban university hospital and regional HIV center may have led to some biases. Among the $17 \mathrm{HIV}$-infected FUO cases in the present study, 10 cases $(58.8 \%)$ were diagnosed as infected with HIV before the transfer to our hospital.

Further prospective studies are necessary to follow FUO patients after hospital discharge and examine the outcomes. In addition, prospective studies should investigate the causes of FUO in patients other than the immunocompetent, such as patients with nosocomial, neutropenic, and HIV-related FUO.

\section{Conclusion}

This study reviewed patients with classic FUO evaluated at our hospital department since it was established. Our study found that, together with the increase in HIV patients in Japan, HIV/AIDS is now the most common cause of FUO. In addition, the percentage of unknown causes of FUO has increased, now exceeding $20 \%$ of cases. As the present study collated data from a long period and reviewed the largest number of FUO cases in Japan to date, these findings will serve as a useful reference for the future care of FUO patients. 
The authors state that they have no Conflict of Interest (COI).

\section{Acknowledgement}

This study received a Grant-in-Aid (S1201013) from the MEXT (Ministry of Education, Culture, Sports, Science and Technology)-Supported Program for the Strategic Research Foundation at Private Universities, 2012-2017.

\section{References}

1. Petersdorf RG, Beeson PB. Fever of unknown origin: report on 100 cases. Medicine 40: 1-30, 1961.

2. Durack DT, Street AC. Fever of unknown origin-reexamined and redefined. Curr Clin Top Infect Dis 11: 35-51, 1991.

3. Alt HL, Barker MH. Fever of unknown origin. JAMA 94: 14571461, 1930.

4. de Kleijn EM, Vandenbroucke JP, van der Meer JW. Fever of unknown origin (FUO). I A. prospective multicenter study of 167 patients with FUO, using fixed epidemiologic entry criteria. The Netherlands FUO Study Group. Medicine (Baltimore) 76: 392400, 1997.

5. Vanderschueren S, Knockaert D, Adriaenssens T, et al. From prolonged febrile illness to fever of unknown origin: the challenge continues. Arch Intern Med 163: 1033-1041, 2003.

6. Miller RF, Hingorami AD, Foley NM. Pyrexia of undetermined origin in patients with human immunodeficiency virus infection and AIDS. Int J STD AIDS 7: 170-175, 1996.

7. Knockaert DC, Vanneste LJ, Bobbaers HJ. Fever of unknown origin in elderly patients. J Am Geriatr Soc 41: 1187-1192, 1993.

8. Zenone T. Fever of unknown origin in adults: evaluation of 144 cases in a non-university hospital. Scand J Infect Dis 38: 632-638, 2006.

9. Bleeker-Rovers CP, Vos FJ, de Kleijn EM, et al. A prospective multicenter study on fever of unknown origin: the yield of a structured diagnostic protocol. Medicine (Baltimore) 86: 26-38, 2007.

10. Colpan A, Onguru P, Erbay A, et al. Fever of unknown origin: analysis of 71 consecutive cases. Am J Med Sci 334: 92-96, 2007.

11. Kucukardali $Y$, Oncul $O$, Cavuslu $S$, et al. The spectrum of diseases causing fever of unknown origin in Turkey: a multicenter study. Int J Infect Dis 12: 71-79, 2008.

12. Cunha BA. Fever of unknown origin: focused diagnostic approach based on clinical clues from the history, physical examination, and laboratory tests. Infect Dis Clin North Am 21: 1137-1187, 2007.

13. Shoji S, Imamura A, Imai Y, et al. Fever of unknown origin: a review of 80 patients from the Shin'etsu area of Japan from 19861992. Intern Med 33: 74-76, 1994.

14. Iikuni Y, Okada J, Kondo H, Kashiwazaki S. Current fever of unknown origin 1982-1992. Intern Med 33: 67-73, 1994.

15. Goto M, Koyama H, Takahashi O, Fukui T. A retrospective review of 226 hospitalized patients with fever. Intern Med 46: 17-22, 2007.

16. Kubota K, Nakamoto Y, Tamaki N, et al. FDG-PET for the diagnosis of fever of unknown origin: a Japanese multi-center study. Ann Nucl Med 25: 355-364, 2011.

17. Nakaya S, Torikai K, Yamazaki Y, Inamura S, Nishisako H. Examination about clinical feature of fever of unknown origin. Journal of St. Marianna 39: 133-141, 2011 (in Japanese).

18. Naito T, Mizooka M, Mitsumoto F, et al. Diagnostic workup for fever of unknown origin: a multicenter collaborative retrospective study. BMJ Open 3: e003971, 2013.

19. Kasai K, Mori M, Hara R, Miyamae T, Imagawa T, Yokota S. National survey of childhood febrile illness cases with fever of unknown origin in Japan. Pediatr Int 53: 421-425, 2011.

20. Ohshima H, Naito T, Kukino J, et al. An analysis of the 215 patients with fever of unknown origin. Juntendo Medical Journal 51: 167-173, 2005 (in Japanese, Abstract in English).

21. Bleeker-Rovers CP, Vos FJ, Mudde AH, et al. A prospective multicenter study of the value of FDG-PET as part of a structured diagnostic protocol in patients with fever of unknown origin. Eur $\mathbf{J}$ Nucl Med Mol Imaging 34: 694-703, 2007.

22. Horowitz HW. Fever of unknown origin or fever of too many origins? N Engl J Med 368: 197-199, 2013.

23. Mourad O, Palda V, Detsky AS. A comprehensive evidence-based approach to fever of unknown origin. Arch Intern Med 163: 545551, 2003.

24. AIDS Prevention Information Network. Available at: http://api-net. jfap.or.jp/status/2012/12nenpo/h24gaiyo.pdf. Accessed November 29, 2013.

25. Zhang J, Chen B, Xu X, et al. Clinical features of 66 lymphoma patients presenting with a fever of unknown origin. Intern Med 51: 2529-2536, 2012.

26. Statistics Bureau, Director-general for policy planning, Statistical research and training institute. Available at: http://www.stat.go.jp/ english/data/jinsui/tsuki/index.htm. Accessed November 29, 2013.

(C) 2014 The Japanese Society of Internal Medicine http://www.naika.or.jp/imonline/index.html 